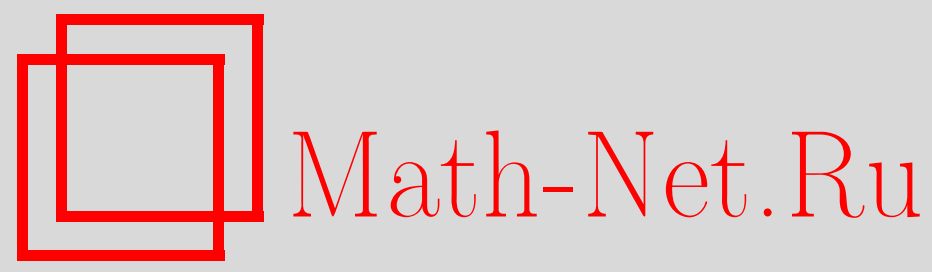

В. А. Смирнов, Биоперады и биалгебры Хопфа в теории кобордизмов, Матем. заметки, 1999, том 65, выпуск 2, 270279

DOI: https://doi.org/10.4213/mzm1049

Использование Общероссийского математического портала Math-Net.Ru подразумевает, что вы прочитали и согласны с пользовательским соглашением http://www . mathnet.ru/rus/agreement

Параметры загрузки:

IP : 54.164 .48 .24

26 апреля 2023 г., 05:17:15

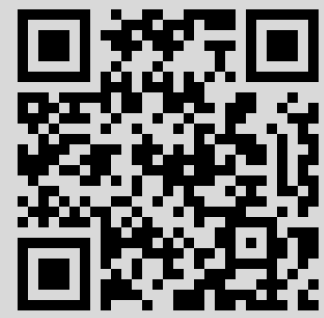


ТОм 65 вЫПУСК 2 фЕВРАЛЬ 1999

УДК 515.142 .42

\section{БИОПЕРАДЫ И БИАЛГЕБРЫ ХОПФА \\ В ТЕОРИИ КОБОРДИЗМОВ}

\section{В. А. Смирнов}

Предлагается конструкция операций Дайера-Лашофа для кобордизмов топологических пространств.

Библиография: 6 названий.

В работе [1] было показано, что операции Стинрода в когомологиях топологического пространства и операции Дайера-Лашофа в гомологиях $E_{\infty}$-пространства индуцируются действием биалгебры Хопфа $E_{*}$, представляющей собой гомологии операды $E$. Здесь мы определим соответствующую биалгебру Хопфа $\mathbb{E}_{*}$ для обобщенных когомологий и, в частности, для кобордизмов топологических пространств. Мы покажем, что кобордизмы топологического пространства и бордизмы $E_{\infty}$-пространства являются алгебрами Хопфа над биалгеброй Хопфа $\mathbb{E}_{*}$. В частности, действие этой алгебры Хопфа задает операции Стинрода на кобордизмах и операции Дайера-Лашофа на бордизмах топологических пространств. Для этого мы будем использовать симплициальные и операдные методы [2].

Симметрическим семейством будем называть семейство $\mathscr{E}=\{\mathscr{E}(n)\}$ топологических пространств $\mathscr{E}(j)$, на которых заданы действия симметрических групा $\Sigma_{n}$. Симметрическое семейство $\mathscr{E}$ будем называть биоперадой, если заданы отображения

$$
\pi: \mathscr{E}(n) \times \mathscr{E}(m) \rightarrow \mathscr{E}(n+m), \quad \gamma: \mathscr{E}(k) \times(\mathscr{E}(n))^{\times k} \rightarrow \mathscr{E}(k n)
$$

удовлетворяющие условиям согласованности с действиями симметрических групп, соотношениям ассоциативности и дистрибутивности [1].

Пусть для краткости $\mathscr{E}(k) * \mathscr{E}(n)=\mathscr{E}(k) \times(\mathscr{E}(n))^{\times k}$. Тогда соотношение ассоциативности означает коммутативность диаграмм

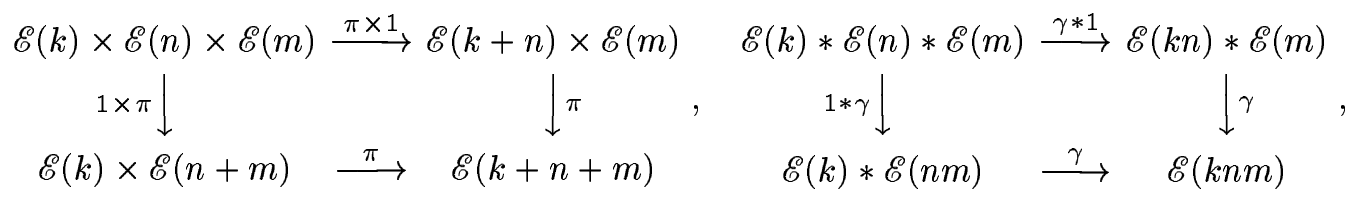

Работа выполнена при финансовой поддержке Английского Королевского Общества. 
а соотношения дистрибутивности означают коммутативность диаграмм

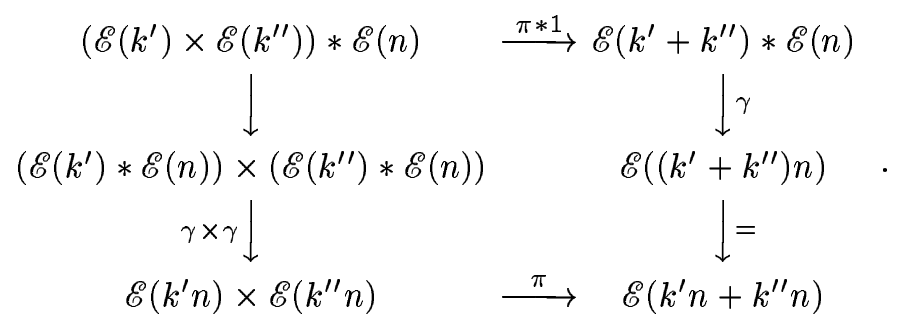

Кроме того, требуется, чтобы диаграммы

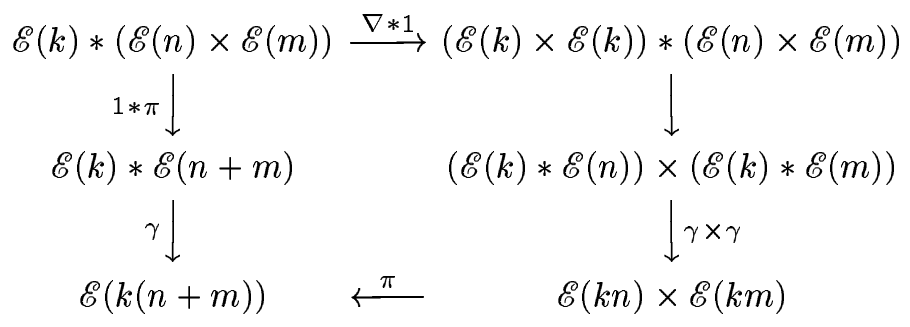

были коммутативны с точностью до некоторых гомеоморфизмов $\xi: \mathscr{E}(k(n+m)) \rightarrow$ $\mathscr{E}(k(n+m))$ таких, что $\xi(x \sigma)=\xi(x) \xi(\sigma), x \in \mathscr{E}(k(n+m)), \sigma \in \Sigma_{k(n+m)}$ (cм. [1]).

Биопераду $E$ будем называть $E_{\infty}$-биоперадой, если пространства $E(n)$ ацикличны и симметрические групшы действуют на них свободно.

Отметим, что понятие биоперады, хотя и близко к понятию операды, тем не менее отличается от него. С одной стороны, для операды $\mathscr{E}$ требуется наличие отображений

$$
\gamma: \mathscr{E}(k) \times \mathscr{E}\left(n_{1}\right) \times \cdots \times \mathscr{E}\left(n_{k}\right) \rightarrow \mathscr{E}\left(n_{1}+\cdots+n_{k}\right)
$$

для различных $n_{1}, \ldots, n_{k}$, а не только для одинаковых, как для биоперады. С другой стороны, для операды не требуется наличие отображений $\pi$, а следовательно, и соотношений дистрибутивности.

Мы так изменили понятие операды, потому что, во-первых, для задания умножений в алгебре Стинрода и Дайера-Лашофа используются только отображения типа $\gamma: \mathscr{E}(k) * \mathscr{E}(n) \rightarrow \mathscr{E}(k n) ;$ во-вторых, для определения соотношений в этих алгебрах, в частности соотношений Адема, требуется задание отображений $\pi: \mathscr{E}(n) \times \mathscr{E}(m) \rightarrow$ $\mathscr{E}(n+m)$ и соотношений дистрибутивности между $\pi$ и $\gamma$.

Конечно, операда $\Sigma=\left\{\Sigma_{n}\right\}$ дает и пример биоперады. Для того чтобы привести пример $E_{\infty}$-биоперады, рассмотрим симплициальные резольвенты $B_{*}\left(*, \Sigma_{n}, \Sigma_{n}\right)$ симметрических групп $\Sigma_{n}$. Тогда их реализации $E(n)$ будут стягиваемыми пространствами, на которых симметрические группы $\Sigma_{n}$ действуют свободно.

Структура биоперады на семействе $\Sigma$ индуцирует соответствующую структуру биоперады на семействе $E$, которое является как операдой, так и биоперадой.

В дальнейшем мы приведем примеры биоперад, не являющихся операдами.

Пусть $\mathscr{F}=\{\mathscr{F}(n)\}-$ семейство топологических пространств, $\mathscr{E}$ - биоперада. Будем говорить, что семейство $\mathscr{F}$ есть $\mathscr{E}$-мультипликативное семейство, если заданы отображения

$$
\pi: \mathscr{F}(n) \times \mathscr{F}(m) \rightarrow \mathscr{F}(n+m), \quad \mu: \mathscr{E}(k) * \mathscr{F}(n) \rightarrow \mathscr{F}(k n)
$$


которые согласованы с действиями симметрических групп и удовлетворяют соотношениям ассоциативности и дистрибутивности.

Пространство $X$ будем назьвать $\mathscr{E}$-мультипликативным пространством, если соответствуюшее семейство $\{X(n)\}, X(n)=X$, является $\mathscr{E}$-мультипликативным.

Если биоперада $E$ является $E_{\infty}$-биоперадой, то $E$-мультипликативное семейство $F$ будем назьвать $E_{\infty}$-мультипликативным семейством, а $E$-мультипликативноепространство $X-E_{\infty}$-мультипликативным пространством.

Пусть $\mathscr{E}=\{\mathscr{E}(n)\}$ - биоперада. Положим $\mathscr{E}_{*}(n)=H_{*}\left(\mathscr{E}(n) / \Sigma_{n}\right)$ и определим $\mathscr{E}_{*}(k) * \mathscr{E}_{*}(n)$ как тензорные произведения $\mathscr{E}_{*}(k) \otimes \mathscr{E}_{*}(n)$, размерность элементов $x \otimes y \in \mathscr{E}_{*}(k) * \mathscr{E}_{*}(n)$ в которых берется равной $\operatorname{dim}(x)+k \operatorname{dim}(y)$. Структура биоперады на $E$ индуцирует произведения

$$
\pi_{*}: \mathscr{E}_{*}(n) \otimes \mathscr{E}_{*}(m) \rightarrow \mathscr{E}_{*}(n+m), \quad \gamma_{*}: \mathscr{E}_{*}(k) * \mathscr{E}_{*}(n) \rightarrow \mathscr{E}_{*}(k n)
$$

Кроме того, имеется копроизведение

$$
\nabla_{*}: \mathscr{E}_{*}(n) \rightarrow \mathscr{E}_{*}(n) \otimes \mathscr{E}_{*}(n)
$$

Из определения биоперады непосредственно следует, что семейство $\mathscr{E}_{*}=\left\{\mathscr{E}_{*}(n)\right\}$ с операциями $\pi_{*}, \gamma_{*}, \nabla_{*}$ является биалгеброй Хопфа [1], т.е. вьполняются следующие условия:

1) операции $\left(\pi_{*}, \nabla_{*}\right)$ и $\left(\gamma_{*}, \nabla_{*}\right)$ определяют структуры алгебр Хопфа на $\mathscr{E}_{*}$;

2 ) между операциями $\gamma_{*}$ и $\pi_{*}$ имеют место соотношения дистрибутивности, означающие коммутативность диаграмм

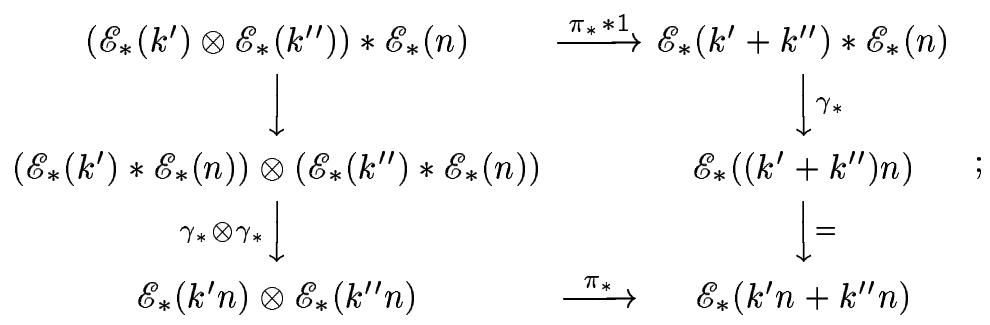

кроме того, диаграммы

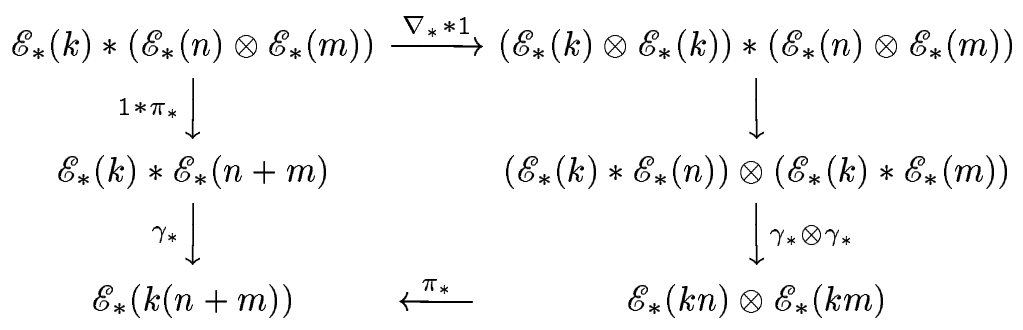

коммутативны с точностью до некоторых изоморфизмов $\xi_{*}: \mathscr{E}_{*}(k(n+m)) \rightarrow$ $\mathscr{E}_{*}(k(n+m))$. 
Заметим, что понятие биалгебры Хопфа близко, но не совпадает с понятием кольца Хопфа [3]. Отличие состоит в соотношениях дистрибутивности и в том, что мы не требуем коммутативности произведения $\gamma_{*}$.

Если $\mathscr{F}=\{\mathscr{F}(n)\}-\mathscr{E}$-мультипликативное семейство, то на гомологиях $\mathscr{F}_{*}=\left\{\mathscr{F}_{*}(n)\right\}$, $\mathscr{F}_{*}(n)=H_{*}(\mathscr{F}(n))$ имеется структура алгебры Хопфа, состоящая из операций

$$
\begin{gathered}
\pi_{*}: \mathscr{F}_{*}(n) \otimes \mathscr{F}_{*}(m) \rightarrow \mathscr{F}_{*}(n+m), \quad \mu_{*}: \mathscr{E}_{*}(k) * \mathscr{F}_{*}(n) \rightarrow \mathscr{F}_{*}(k n), \\
\nabla_{*}: \mathscr{F}_{*}(n) \rightarrow \mathscr{F}_{*}(n) \otimes \mathscr{F}_{*}(n),
\end{gathered}
$$

которые удовлетворяют условиям, аналогичным условиям 1), 2) в определении биалгебры Хопфа.

Заметим, что если $E-E_{\infty}$-биоперада и $F-E$-мультипликативное семейство, то задание действия $\mu_{*}: E_{*} * F_{*} \rightarrow F_{*}$ равносильно заданию на $F_{*}$ действия алгебры Дайера-Лашофа.

Пусть $\mathbb{F}$ - поле действительных чисел $\mathbb{R}$, или поле комплексных чисел $\mathbb{C}$, или тело кватернионов $\mathbb{H}$. Рассмотрим семейство $\mathrm{GL}=\{\mathrm{GL}(\mathbb{F}, n)\}_{n \geqslant 1}$ полных линейньх групп $\mathrm{GL}(\mathbb{F}, n)$ векторных пространств $\mathbb{F}^{n}$. Перестановка сомножителей в $\mathbb{F}^{n}$ индуцирует свободное действие $\Sigma_{n}$ на $\mathrm{GL}(\mathbb{F}, n)$ :

$$
(\sigma f)(x)=f\left(\sigma^{-1} x\right), \quad f \in \mathrm{GL}(\mathbb{F}, n), \quad x \in \mathbb{F}^{n}, \quad \sigma \in \Sigma_{n}
$$

Представляя элементы из $\mathrm{GL}(\mathbb{F}, n)$ матрицами, получим, что действие симметрических групп $\Sigma_{n}$ определяется перестановками столбцов этих матриц.

Имеют место отображения $\pi: \mathrm{GL}(\mathbb{F}, n) \times \mathrm{GL}(\mathbb{F}, m) \rightarrow \mathrm{GL}(\mathbb{F}, n+m)$, которые сопоставляют паре матрищ $(A, B)$ матрицу

$$
A \oplus B=\left(\begin{array}{cc}
A & O \\
O & B
\end{array}\right),
$$

где $O$ - нулевая матрица.

Определим отображения $\gamma: \mathrm{GL}(\mathbb{F}, k) * \mathrm{GL}(\mathbb{F}, n) \rightarrow \mathrm{GL}(\mathbb{F}, k n)$. Пусть $A \in \mathrm{GL}(\mathbb{F}, k)$, $B_{i} \in \mathrm{GL}(\mathbb{F}, n), 1 \leqslant i \leqslant k$,

$$
A=\left(\begin{array}{ccc}
a_{11} & \ldots & a_{1 k} \\
\ldots \ldots & \ldots & \ldots \\
a_{k 1} & \ldots & a_{k k}
\end{array}\right) .
$$

Обозначим через $A(n)$ матрицу в $\mathrm{GL}(\mathbb{F}, k n)$, состоящую из $(n \times n)$-матриц

$$
\left(\begin{array}{cccc}
a_{i j} & 0 & \ldots & 0 \\
0 & a_{i j} & \ldots & 0 \\
\ldots & \ldots & \ldots & \ldots \\
0 & 0 & \ldots & a_{i j}
\end{array}\right), \quad 1 \leqslant i, j \leqslant k
$$

Используя тензорное произведение матриц, запишем $A(n)$ в виде $A(n)=A \otimes I_{n}$, где $I_{n}-$ единичная матрища в $\mathrm{GL}(\mathbb{F}, n)$.

Положим $\gamma\left(A \times B_{1} \times \cdots \times B_{k}\right)=A(n) \cdot\left(B_{1} \oplus \cdots \oplus B_{k}\right)$. Прямые вычисления показывают, что выполняются требуемые соотношения и, следовательно, имеет место 
ПРЕДЛОЖЕНИЕ 1. Семейство $\mathrm{GL}=\{\mathrm{GL}(\mathbb{F}, n)\}$ с определенными выше операииям и

$$
\pi: \mathrm{GL}(\mathbb{F}, n) \times \mathrm{GL}(\mathbb{F}, m) \rightarrow \mathrm{GL}(\mathbb{F}, n+m), \quad \gamma: \mathrm{GL}(\mathbb{F}, k) * \mathrm{GL}(\mathbb{F}, n) \rightarrow \mathrm{GL}(\mathbb{F}, k n)
$$

является биоперадой. В частности, биоперадами являются семейства ортогональных әрупп $\mathrm{O}=\{\mathrm{O}(n)\}$, унитарных групп $\mathrm{U}=\{\mathrm{U}(n)\}$ и симплектических групп $\mathrm{Sp}=\{\mathrm{Sp}(n)\}$.

Для групп $G(n)=\mathrm{GL}(\mathbb{F}, n)$ рассмотрим пространства $E G(n)$, являющиеся реализациями симплициальных резольвент

$$
B_{*}(*, G(n), G(n)): G(n) \leftarrow G(n) \times G(n) \leftarrow \cdots \leftarrow G(n)^{\times m+1} \leftarrow \cdots .
$$

Структура биоперады на GL индуцирует структуру биоперады на $E G=\{E G(n)\}$. Учитьвая, что пространства $E(n)$ ацикличны со свободным действием симметрических групп, получаем

ПРЕДЛОЖЕНИЕ 2. Биоперада $E G$ является $E_{\infty}$-биоперадой. В частности, $E_{\infty}$ биоперадами являются $E \mathrm{O}=\{E \mathrm{O}(n)\}, E \mathrm{U}=\{E \mathrm{U}(n)\}$ и $E \mathrm{Sp}=\{E \mathrm{Sp}(n)\}$.

Заметим, что представления симметрических групा $\Sigma_{n} \rightarrow \mathrm{GL}(\mathbb{F}, n)$ определяют отображение биоперад $E \rightarrow E G$, индуцирующее изоморфизм гомологий $E_{*} \rightarrow E G_{*}$. В том числе имеют место изоморфизмы биалгебр Хопфа $E_{*} \cong E \mathrm{O}_{*} \cong E \mathrm{U}_{*} \cong E \mathrm{Sp}_{*}$.

Для групा $G(n)=\mathrm{GL}(\mathbb{F}, n)$ рассмотрим классифицирующие пространства $B G(n)$, являющиеся реализациями симплищиальных объектов

$$
B_{*}(*, G(n), *): * \leftarrow G(n) \leftarrow G(n) \times G(n) \leftarrow \cdots \leftarrow G(n)^{\times m+1} \leftarrow \cdots .
$$

Структура биоперады на GL индуцирует на $B G=\{B G(n)\}$ действие биоперады $E G$, т.е. имеет место

ПРЕДЛОЖЕНИЕ 3. Семейство ВG является ЕG-мультипликативным семейством. В частности, ВО есть ЕО-мультипликативное семейство, ВU есть ЕU-мультипликативное семейство, BSp есть ESp-мультипликативное семейство. Следовательно, гомологии $B \mathrm{O}_{*}=\left\{H_{*}(B \mathrm{O}(n))\right\}, B \mathrm{U}_{*}=\left\{H_{*}(B \mathrm{U}(n))\right\}$, $B \mathrm{Sp}_{*}=\left\{H_{*}(B \mathrm{Sp}(n))\right\}$ являются алгебрами Хопфа над биалгеброй Хопфа $E_{*}$.

Перейдем теперь к спектрам. Пусть $\mathscr{E}-$ биоперада. Мультипликативный спектр $\mathbb{X}=\{\mathbb{X}(n)\}$ будем назьвать $\mathscr{E}$-мультипликативным, если заданы операции $\mu: \mathscr{E}(k) *$ $\mathbb{X}(n) \rightarrow \mathbb{X}(k n)$, которые вместе с мультипликативной структурой на $\mathbb{X}$ образуют структуру $\mathscr{E}$-мультипликативного семейства. Если $E$ является $E_{\infty}$-биоперадой, то $E$-мультипликативньй спектр $\mathbb{X}=\{\mathbb{X}(n)\}$ будем называть $E_{\infty}$-мультипликативным спектром.

Заметим, что понятие $E_{\infty}$-мультипликативного спектра по существу совпадает с понятием $H_{\infty}$-кольцевого предспектра [4] и отличается от понятий $H_{\infty}$ - и $E_{\infty}$-кольцевых спектров [5] тем, что мы не требуем согласованности операций $\mu$ со структурой спектра на $\mathbb{X}$.

Пусть $\mathbb{S}=\left\{S^{n}\right\}$ - спектр сфер. Действиегрупш $\mathrm{O}(n)$ на пространствах $\mathbb{R}^{n}$ определяет действие групп $\mathrm{O}(n)$ на $S^{n}$. Рассмотрим симплициальный объект

$$
B_{*}\left(*, \mathrm{O}(n), S^{n}\right): S^{n} \leftarrow \mathrm{O}(n) \lambda S^{n} \leftarrow \cdots \leftarrow \mathrm{O}(n)^{\times m} \lambda S^{n} \leftarrow \cdots,
$$


где " $\lambda$ " обозначает полупрямоепроизведение. Его реализация является пространством Тома $M \mathrm{O}(n)$, и семейство $M \mathrm{O}=\{M \mathrm{O}(n)\}-$ спектром Тома. Аналогично, имеются спектры Тома $M \mathrm{U}, M \mathrm{Sp}$.

Так же, как была определена структура операды на семействе GL $=\{\mathrm{GL}(n)\}$, определяются действия $\mu: \mathrm{O}(k) \times\left(\mathbb{R}^{n}\right)^{\times k} \rightarrow \mathbb{R}^{k n}$, которые индуцируют действия биоперады $\mathrm{O}=\{\mathrm{O}(n)\}$ на семействе сфер $\mathbb{S}=\left\{S^{n}\right\}$. Кроме того, имеются естественные отображения $\pi: S^{n} \wedge S^{m} \rightarrow S^{n+m}$. Непосредственная проверка показьвает, что эти отображения $\mu$ и $\pi$ удовлетворяют требуемым соотношениям и, следовательно, спектр сфер $\mathbb{S}=\left\{S^{n}\right\}$ является О-мультипликативньг.

Эта структура на $\mathbb{S}$ индуцирует отображения

$$
\pi: M \mathrm{O}(n) \wedge M \mathrm{O}(m) \rightarrow M \mathrm{O}(n+m), \quad \mu: E \mathrm{O}(k) * M \mathrm{O}(n) \rightarrow M \mathrm{O}(k n)
$$

которые определяют на спектре Тома $M \mathrm{O}=\{M \mathrm{O}(n)\}$ структуру $E \mathrm{O}-$ мультипликативного спектра. Аналогичная структура имеется и на других спектрах Тома, т.е. имеет место

ПРЕДЛОЖЕНИЕ 4. Спектр Тома $М \mathrm{O}$ является ЕО-мультипликативным спектром, $M \mathrm{U}$ является ЕU-мультипликативным спектром и $M \mathrm{Sp}$ является $E \mathrm{Sp}$ мультипликативным спектром. Следовательно, гомологии $\mathrm{MO}_{*}=\left\{H_{*}(\mathrm{MO}(n))\right\}$, $M \mathrm{U}_{*}=\left\{H_{*}(M \mathrm{U}(n))\right\}, M \mathrm{Sp}_{*}=\left\{H_{*}(M \mathrm{Sp}(n))\right\}$ являются алгебрами Хопфа над биалгеброй Хопфа $E_{*}$.

Рассмотрим теорию гомологий, ассоциированную с мультипликативным спектром $\mathbb{H}$. Для пространства $X$ гомологии $\mathbb{H}_{*}(X)=\left\{\mathbb{H}_{n}(X)\right\}$ определяются формулой

$$
\mathbb{H}_{n}(X)=\lim _{i} \pi_{n+i}(\mathbb{H}(i) \wedge X),
$$

где предел берется относительно гомоморфизмов

$$
\pi_{n+i}(\mathbb{H}(i) \wedge X) \rightarrow \pi_{n+i+1}(S \mathbb{H}(i) \wedge X) \rightarrow \pi_{n+i+1}(\mathbb{H}(i+1) \wedge X)
$$

В частности, имеют место теории гомологий $\mathbb{M O}_{*}, \mathbb{M U}_{*}, \mathbb{M S p}_{*}$.

Пусть теперь $\mathbb{X}$ - коспектр, т.е. семейство $\mathbb{X}=\{\mathbb{X}(m)\}$ пространств $\mathbb{X}(m)$ с заданными отображениями $\mathbb{X}(m) \rightarrow S \mathbb{X}(m-1)$. Тогда гомологии коспектра $\mathbb{X}$ определяются формулой

$$
\mathbb{H}_{n}(\mathbb{X})=\lim _{m} \mathbb{H}_{n+m}(\mathbb{X}(m)),
$$

где предел берется относительно гомоморфизмов

$$
\mathbb{H}_{n+m}(\mathbb{X}(m)) \rightarrow \mathbb{H}_{n+m}(S \mathbb{X}(m-1)) \rightarrow \mathbb{H}_{n+m-1}(\mathbb{X}(m-1))
$$

Легко видеть, что если $X$ - топологическое пространство и $\mathbb{S} X-$ надстроечньй коспектр, то гомологии этого коспектра будут изоморфны гомологиям пространства $X$.

Мы обобщим понятие коспектра и введем понятие $\mathbb{H}$-коспектра. А именно, семейство $\mathbb{X}=\{\mathbb{X}(m)\}$ пространств $\mathbb{X}(m)$ будем называть $\mathbb{H}-$ коспектром, если для любого $m$ задано отображение $\varphi: \mathbb{X}(m) \rightarrow \mathbb{H}(k) \wedge \mathbb{X}(m-k)$.

Ясно, что $\mathbb{S}$-коспектры есть просто коспектры. Для того чтобы привести нетривиальные примеры $\mathbb{H}-$ коспектров, рассмотрим биопераду $E$ и $E$-мультипликативный спектр $\mathbb{H}$. Для коспектра $\mathbb{X}$ положим $E(k, \mathbb{X}(m))=E(k) *_{\Sigma_{k}} \mathbb{X}(m)$. Пусть $E(k, \mathbb{X})-$ 
семейство пространств $\{E(k, \mathbb{X})(n)\}$, для которых $E(k, \mathbb{X})(k m)=E(k, \mathbb{X}(m))$, если $n=k m$, и надстройки над этими пространствами для других $n$.

Отображения

$$
\begin{aligned}
E(k, \mathbb{X}(m)) & \rightarrow E(k, S \mathbb{X}(m-1)) \rightarrow E(k, \mathbb{H}(1) \wedge \mathbb{X}(m-1)) \\
& \rightarrow(E(k, \mathbb{H}(1)) \wedge(E(k, \mathbb{X}(m-1)) \rightarrow \mathbb{H}(k) \wedge(E(k, \mathbb{X}(m-1))
\end{aligned}
$$

определяют на семействе $E(k, \mathbb{X})$ искомую структуру $\mathbb{H}$ коспектра. В частности, для сферического коспектра $\mathbb{S}$ мы имеем $\mathbb{H}$-коспектр $E(k, \mathbb{S})$, которьй играет важную роль при построении операций Стинрода и Дайера-Лашофа.

Пусть $\mathbb{X}-\mathbb{H}$-коспектр, $\varphi: \mathbb{X}(m) \rightarrow \mathbb{H}(k) \wedge \mathbb{X}(m-k)$. Определим гомологии $\mathbb{H}_{*}^{\varphi}(\mathbb{X})$, которые будем назьвать скрученными гомологиями:

$$
\mathbb{H}_{n}(\mathbb{X})=\lim _{m} \mathbb{H}_{n+m}(\mathbb{X}(m)),
$$

где предел берется относительно гомоморфизмов

$$
\varphi_{*}: \mathbb{H}_{n+m}(\mathbb{X}(m)) \rightarrow \mathbb{H}_{n+m}(\mathbb{H}(k) \wedge \mathbb{X}(m-k)) \rightarrow \mathbb{H}_{n+m-k}(\mathbb{X}(m-k)) \text {. }
$$

В частности, определены скрученные гомологии

$$
\mathbb{H}_{n}^{\varphi}(E(k, \mathbb{S}))=\lim _{m} \mathbb{H}_{n+k m}\left(E\left(k, S^{m}\right)\right),
$$

где предел берется относительно гомоморфизмов

$$
\varphi_{*}: \mathbb{H}_{n+k m}\left(E\left(k, S^{m}\right)\right) \rightarrow \mathbb{H}_{n+k(m-1)}\left(E\left(k, S^{m-1}\right)\right) .
$$

Ясно, что скрученные гомологии коспектра, рассматриваемого как $\mathbb{H}$-коспектр, изоморфны просто гомологиям коспектра.

Пусть $E$ - биоперада, $X-E$-мультипликативное пространство, $\mathbb{H}-E$-мультипликативньй спектр. Наша цель состоит в построении операций на гомологиях $\mathbb{H}_{*}(X)$, аналогичных операциям Дайера-Лашофа для обычных гомологий.

Отметим, что в работах [4], [5] предложен общий метод построения операций Дайера-Лашофа для теорий гомологий, соответствуюших $H_{\infty}$ и $E_{\infty}$-кольцевым спектрам. Переход от $H_{\infty}$-предспектров к $H_{\infty}$-кольцевым спектрам осуществляется с помощью некоторого стабилизируюшего функтора $Z$. Мы определим операции Дайера-Лашофанепосредственно для $E$-мультипликативных спектров в том числе и для $H_{\infty}$-предспектров. Это позволит определить не только операции Дайера-Лашофа для обобщенных гомологий, но и саму алгебру Дайера-Лашофа. Для этого и потребуется понятие скрученных гомологий.

Пусть $x \in \mathbb{H}_{n}(X)$ и $f: S^{n+m} \rightarrow \mathbb{H}(m) \wedge X$ - представляющее его непрерьвное отображение. Рассмотрим отображение

$$
E(k, f): E\left(k, S^{n+m}\right) \rightarrow E(k, \mathbb{H}(m) \wedge X)
$$

и возьмем композицию отображений (1) и

$$
E(k, \mathbb{H}(m) \wedge X) \rightarrow E(k, \mathbb{H}(m)) \wedge E(k, X) \rightarrow \mathbb{H}(k m) \wedge X
$$


Получим отображение $E\left(k, S^{n+m}\right) \rightarrow \mathbb{H}(k m) \wedge X$ и, следовательно, отображение

$$
\mathbb{H}(j) \wedge E\left(k, S^{n+m}\right) \rightarrow \mathbb{H}(j) \wedge \mathbb{H}(k m) \wedge X \rightarrow \mathbb{H}(j+k m) \wedge X
$$

которое индуцирует гомоморфизм гомотопических групш

$$
\pi_{i+j+k(n+m)}\left(\mathbb{H}(j) \wedge E\left(k, S^{n+m}\right)\right) \rightarrow \pi_{i+j+k(n+m)}(\mathbb{H}(j+k m) \wedge X) .
$$

Переходя к пределу по $j$, получим гомоморфизм $\mu_{f}: \mathbb{H}_{i+k(n+m)}\left(E\left(k, S^{n+m}\right)\right) \rightarrow$ $\mathbb{H}_{i+k n}(X)$ и, следовательно, действие

$$
\mu_{*}: \mathbb{H}_{i+k(n+m)}\left(E\left(k, S^{n+m}\right)\right) \times \pi_{n+m}(\mathbb{H}(m) \wedge X) \rightarrow \mathbb{H}_{i+k n}(X) .
$$

Для надстроечного гомоморфизма $S: \pi_{n+m}(\mathbb{H}(m) \wedge X) \rightarrow \pi_{n+m+1}(\mathbb{H}(m+1) \wedge X)$ имеют место коммутативные диаграммы

$$
\begin{aligned}
& \mathbb{H}_{i+k(n+m+1)}\left(E\left(k, S^{n+m+1}\right)\right) \times \pi_{n+m+1}(\mathbb{H}(m+1) \wedge X) \stackrel{\mu_{*}}{\longrightarrow} \mathbb{H}_{i+k n}(X) \\
& 1 \times S \uparrow \\
& \mathbb{H}_{i+k(n+m+1)}\left(E\left(k, S^{n+m+1}\right)\right) \times \pi_{n+m}(\mathbb{H}(m) \wedge X) \\
& \varphi_{*} \times 1 \downarrow \\
& \mathbb{H}_{i+k(n+m)}\left(E\left(k, S^{n+m}\right)\right) \times \pi_{n+m}(\mathbb{H}(m) \wedge X) \quad \stackrel{\mu_{*}}{\longrightarrow} \mathbb{H}_{i+k n}(X)
\end{aligned}
$$

Переходя к пределу по $m$, получим требуемое действие

$$
\mu_{*}: \mathbb{H}_{i}^{\varphi}(E(k, \mathbb{S})) \times \mathbb{H}_{n}(X) \rightarrow \mathbb{H}_{i+n k}(X),
$$

которое аддитивно по первому сомножителю и, вообще говоря, неаддитивно по второму.

Обозначим через $\mathbb{H}_{*}^{\varphi}(E(k, \mathbb{S})) * \mathbb{H}_{*}(X)$ соответствующее полутензорное произведение, размерность элементов $e_{i} * x_{n}$ в котором считается равной $i+k n$. Тогда операции $\mu_{*}: \mathbb{H}_{*}^{\varphi}(E(k, \mathbb{S})) * \mathbb{H}_{*}(X) \rightarrow \mathbb{H}_{*}(X)$ сохраняют размерность.

Если в определении операций Дайера-Лашофа $\mathbb{H}_{*}^{\varphi}(E(k, \mathbb{S})) * \mathbb{H}_{*}(X) \rightarrow \mathbb{H}_{*}(X)$ вместо пространства $X$ мы возьмем $E\left(n, S^{m}\right)$, то получим отображения

$$
\gamma_{*}: \mathbb{H}_{*}^{\varphi}(E(k, \mathbb{S})) * \mathbb{H}_{*}\left(E\left(n, S^{m}\right)\right) \rightarrow \mathbb{H}_{*}\left(E\left(k n, S^{m}\right)\right) .
$$

Переходя к пределу по $m$, получим операции

$$
\gamma_{*}: \mathbb{H}_{*}^{\varphi}(E(k, \mathbb{S})) * \mathbb{H}_{*}^{\varphi}(E(n, \mathbb{S})) \rightarrow \mathbb{H}_{*}^{\varphi}(E(k n, \mathbb{S})) .
$$

Полагая $\mathbb{E}_{*}(n)=\mathbb{H}_{*}^{\varphi}(E(n, \mathbb{S}))$, получим операции $\gamma_{*}: \mathbb{E}_{*}(k) * \mathbb{E}_{*}(n) \rightarrow \mathbb{E}_{*}(k n)$.

Из определения операды $E$ непосредственно следует, что построенные операции $\gamma_{*}$ ассоциативны и, следовательно, семейство $\mathbb{E}_{*}=\left\{\mathbb{E}_{*}(n)\right\}, \mathbb{E}_{*}(n)=\mathbb{H}_{*}^{\varphi}(E(n, \mathbb{S}))$, можно считать алгеброй с операцией $\gamma_{*}: \mathbb{E}_{*}(k) * \mathbb{E}_{*}(n) \rightarrow \mathbb{E}_{*}(k n)$. Если $X$ является $E$-мультипликативньгм пространством, то операции $\mu_{*}: \mathbb{E}_{*}(k) * \mathbb{H}_{*}(X) \rightarrow \mathbb{H}_{*}(X)$ индуцируют действие алгебры $\mathbb{E}_{*}$ на гомологиях $\mathbb{H}_{*}(X)$.

На $\mathbb{E}_{*}=\left\{\mathbb{E}_{*}(n)\right\}$ имеется дополнительная структура модуля над алгеброй $\mathbb{H}_{*}\left(S^{0}\right)$, $\mu_{*}: \mathbb{H}_{*}\left(S^{0}\right) \otimes \mathbb{E}_{*}(n) \rightarrow \mathbb{E}_{*}(n)$. Так как $S^{0}$ можно считать тривиальным $E$-мультипликативным пространством, имеется действие $\mu_{*}: \mathbb{E}_{*}(k) * \mathbb{H}_{*}\left(S^{0}\right) \rightarrow \mathbb{H}_{*}\left(S^{0}\right)$. Кроме того, произведения $\pi: E(n) \times E(m) \rightarrow E(n+m)$ индуцируют произведения

$$
\pi_{*}: \mathbb{E}_{*}(n) \otimes \mathbb{E}_{*}(m) \rightarrow \mathbb{E}_{*}(n+m) .
$$

Диагональ $\nabla: E(n) \rightarrow E(n) \times E(n)$ в случае, когда $\mathbb{E}_{*}(n)$ является плоским $\mathbb{H}_{*}\left(S^{0}\right)$ модулем, индуцирует копроизведение $\nabla_{*}: \mathbb{E}_{*}(n) \rightarrow \mathbb{E}_{*}(n) \otimes_{\mathbb{H}_{*}\left(S^{0}\right)} \mathbb{E}_{*}(n)$.

Между этими операциями имеются все возможные соотношения ассоциативности, дистрибутивности и соотношения Хопфа. Мы будем называть такую структуру на $\mathbb{E}_{*}=$ $\left\{\mathbb{E}_{*}(n)\right\}$ структурой $\mathbb{H}_{*}\left(S^{0}\right)$-биалгебры Хопфа. Таким образом, имеем 
ПРЕДЛОЖЕНИЕ 5. Если $E$ - биоперада $и \mathbb{H}-$ - -мультипликативный спектр, то гомологии $\mathbb{E}_{*}=\left\{\mathbb{E}_{*}(n)\right\}, \mathbb{E}_{*}(n)=\mathbb{H}_{*}^{\varphi}(E(n, \mathbb{S}))$, являются $\mathbb{H}_{*}\left(S^{0}\right)$-биалгеброй Хопфа. Если $X$ - Е-мультипликативное пространство, то гомологии $\mathbb{H}_{*}(X)$ являются $\mathbb{H}_{*}\left(S^{0}\right)$-алгеброй над $\mathbb{H}_{*}\left(S^{0}\right)$-биалгеброй Хопфа $\mathbb{E}_{*}$.

Заметим, что для определения операций Дайера-Лашофа с $\mathbb{Z} / p$-коэффициентами $\mathbb{H}_{*}(\mathbb{X} ; \mathbb{Z} / p)$ необходимо лиш заменить спектр сфер $\mathbb{S}$ на соответствуюший спектр Мура $\mathbb{S}_{(p)}[5]$. В этом случае мы получим алгебру $\mathbb{E}_{*}^{(p)}=\left\{\mathbb{E}_{*}^{(p)}(n)=\mathbb{H}_{*}^{\varphi}\left(E\left(k, \mathbb{S}_{(p)}\right) ; \mathbb{Z} / p\right)\right\}$ и операции $\mu_{*}: \mathbb{E}_{*}^{(p)}(k) * \mathbb{H}_{*}(X ; \mathbb{Z} / p) \rightarrow \mathbb{H}_{*}(X ; \mathbb{Z} / p)$.

Рассмотрим теперь теорию когомологий $\mathbb{H}^{*}$, ассоциированную со спектром $\mathbb{H}$. Если $X$ - топологическое пространство, то гомологии $\mathbb{H}^{*}(X)=\left\{\mathbb{H}^{n}(X)\right\}$ определяются формулой

$$
\mathbb{H}^{n}(X)=\lim _{m} \pi_{i}(X, \mathbb{H}(n+i)),
$$

где предел берется относительно гомоморфизмов

$$
\pi_{i}(X, \mathbb{H}(n+i)) \rightarrow \pi_{m+1}(X, S \mathbb{H}(n+i)) \rightarrow \pi_{m+1}(X, \mathbb{H}(n+i+1))
$$

Определим также когомологии коспектра $\mathbb{X}=\{\mathbb{X}(m)\}$ :

$$
\mathbb{H}^{n}(\mathbb{X})=\lim _{m} \mathbb{H}^{n+m}(\mathbb{X}(m)),
$$

где предел берется относительно гомоморфизмов

$$
\mathbb{H}^{n+m-1}(\mathbb{X}(m-1)) \rightarrow \mathbb{H}^{n+m}(S \mathbb{X}(m-1)) \rightarrow \mathbb{H}^{n+m}(\mathbb{X}(m))
$$

Ясно, что когомологии надстроечного коспектра $\mathbb{S} \wedge X=\left\{S^{n} X\right\}$ над пространством $X$ изоморфны когомологиям пространства $X$.

Для $\mathbb{H}$-коспектра $\mathbb{X}$ определим скрученные когомологии:

$$
\mathbb{H}_{\varphi}^{n}(\mathbb{X})=\lim _{m} \mathbb{H}^{n+m}(\mathbb{X}(m)),
$$

где предел берется относительно гомоморфизмов

$$
\mathbb{H}^{n+m-k}(\mathbb{X}(m-k)) \rightarrow \mathbb{H}^{n+m}(\mathbb{H}(k) \wedge \mathbb{X}(m-k)) \rightarrow \mathbb{H}^{n+m}(\mathbb{X}(m)) .
$$

Ясно, что скрученные когомологии коспектра $\mathbb{X}$, рассматриваемого как $\mathbb{H}-$ коспектр по отношению к отображениям $\mathbb{X}(m) \rightarrow S \mathbb{X}(m-1) \rightarrow \mathbb{H}(1) \wedge \mathbb{X}(m-1)$, изоморфны когомологиям коспектра $\mathbb{X}$.

Пусть $E$ - биоперада, $\mathbb{H}-E$-мультипликативный спектр, $X$ - топологическое пространство. Наша цель состоит в определении операций на когомологиях $\mathbb{H}^{*}(X)$ в том числе и на кобордизмах, аналогичных операциям Стинрода [6].

Пусть $x \in \mathbb{H}^{n}(X)$ и $f: S^{m} X \rightarrow \mathbb{H}(n+m)$ - представляющее его отображение. Это отображение индуцирует отображение

$$
E\left(k, S^{m} X\right) \rightarrow E(k, \mathbb{H}(n+m)) .
$$

Композиция отображений $(2)$ и $\gamma: E(k, \mathbb{H}(n+m)) \rightarrow \mathbb{H}(k n+k m)$ дает отображение

$$
E\left(k, S^{m} X\right) \rightarrow \mathbb{H}(k n+k m) .
$$


Диагональное отображение $X \rightarrow X \wedge \cdots \wedge X$ индуцирует отображение $E\left(k, S^{m}\right) \wedge X \rightarrow$ $E\left(k, S^{m} X\right)$, композиция которого с (3) дает отображение $E\left(k, S^{m}\right) \wedge X \rightarrow \mathbb{H}(k n+k m)$. Умножая его на $\mathbb{H}(j)$, получим отображение

$$
\mathbb{H}(j) \wedge E\left(k, S^{m}\right) \wedge X \rightarrow \mathbb{H}(j) \wedge \mathbb{H}(k n+k m) \rightarrow \mathbb{H}(j+k n+k m)
$$

и, следовательно, отображение $\mathbb{H}(j) \wedge E\left(k, S^{m}\right) \rightarrow\{X ; \mathbb{H}(j+k n+k m)\}$, которое индуцирует гомоморфизм гомотопических групा

$$
\pi_{i+j+k m}\left(\mathbb{H}(j) \wedge E\left(k, S^{m}\right)\right) \rightarrow \pi_{i+j+k m}(X ; \mathbb{H}(j+k n+k m)) .
$$

Переходя к пределу по $j$, мы получим гомоморфизм $\mu_{f}: \mathbb{H}_{i+k m}\left(E\left(k, S^{m}\right)\right) \rightarrow \mathbb{H}^{k n-i}(X)$. Для надстройки $S f: S^{m+1} X \rightarrow \mathbb{H}(n+m+1)$ имеет место коммутативная диаграмма

$$
\begin{array}{cc}
\mathbb{H}_{i+k(m+1)}\left(E\left(k, S^{m+1}\right)\right) & \stackrel{\mu_{S f}}{\longrightarrow} \mathbb{H}^{k n-i}(X) \\
\varphi_{*} \downarrow & \downarrow= \\
\mathbb{H}_{i+k m}\left(E\left(k, S^{m}\right)\right) & \stackrel{\mu_{f}}{\longrightarrow} \mathbb{H}^{k n-i}(X)
\end{array}
$$

Переходя к пределу по $m$, мы получим требуемое действие

$$
\mu_{*}: \mathbb{H}_{i}^{\varphi}(E(k, \mathbb{S})) \times \mathbb{H}^{n}(X) \rightarrow \mathbb{H}^{k n-i}(X),
$$

которое аддитивно по первому сомножителю и, вообще говоря, неаддитивно по второму.

Обозначим через $\mathbb{E}_{*}(k) * \mathbb{H}^{*}(X)$ соответствующее полутензорное произведение, размерность элементов $e_{i} * x_{n}$ в котором берется равной $k n-i$. Тогда действие $\mu_{*}: \mathbb{E}_{*}(k) *$ $\mathbb{H}^{*}(X) \rightarrow \mathbb{H}^{*}(X)$ сохраняет размерность.

ПРЕДЛОЖЕНИЕ 6. Если E - биоперада $и \mathbb{H}-$ - -мультипликативный спектр, то на когомология $\mathbb{H}^{*}(X)$ топологического пространства $X$ имеется естественная структура $\mathbb{H}^{*}\left(S^{0}\right)$-алгебры над биалгеброй Хопфа $\mathbb{E}_{*}$. В частности, такие структуры имеются на кобордизмах топологических пространств.

Заметим, что для определения операций $\mu_{*}$ на когомологиях с коэффициентами в $\mathbb{Z} / p$ необходимо лишь заменить спектр сфер на соответствуюший спектр Мура $\mathbb{S}_{(p)}$. При этом мы получим операции $\mu_{*}: \mathbb{E}_{*}^{(p)}(k) * \mathbb{H}^{*}(\mathbb{X} ; \mathbb{Z} / p) \rightarrow \mathbb{H}^{*}(\mathbb{X} ; \mathbb{Z} / p)$.

Автор выражает благодарность профессору Манчестерского университета Н. Рэю за помощь в работе.

\section{СПИСОК ЦИТИРОВАННОЙ ЛИТЕРАТУРЫ}

[1] Смирнов В.А. Вторичные операции в гомологиях операды $E$ // Изв. РАН. 1992. Т. 56. №2. C. 449-468.

[2] May J. P. The Geometry of Iterated Loop Spaces. Lecture Notes in Math. V. 271. Berlin: Springer, 1972.

[3] Ravenel D.C., Wilson W.S. The Hopf ring for complex cobordism // J. Pure Appl. Algebra. 1977. V. 9. P. 241-280.

[4] Bruner R., May J. P., McCluer J. E., Steinberger M. $H_{\infty}$ Ring Spectra and their Applications. Lecture Notes in Math. V. 1176. Berlin: Springer, 1986.

[5] May J. P., Quinn F., Ray N., Tornehaive J. $E_{\infty}$-Ring Space and $E_{\infty}$-Ring Spectra. Lecture Notes in Math. V. 577. Berlin: Springer, 1977.

[6] Dieck T. Steenrod-Operationen in Kobordism-Theorien // Math. Z. 1968. V. 107. P. 380-401. 